\title{
Best Possible Bounds for Neuman-Sándor Mean by the Identric, Quadratic and Contraharmonic Means
}

\author{
Tie-Hong Zhao, ${ }^{1}$ Yu-Ming $\mathrm{Chu}^{2}{ }^{2}$ Yun-Liang Jiang, ${ }^{3}$ and Yong-Min $\mathrm{Li}^{4}$ \\ ${ }^{1}$ Department of Mathematics, Hangzhou Normal University, Hangzhou 310036, China \\ ${ }^{2}$ School of Mathematics and Computation Sciences, Hunan City University, Yiyang 413000, China \\ ${ }^{3}$ School of Information \& Engineering, Huzhou Teachers College, Huzhou 313000, China \\ ${ }^{4}$ School of Automation, Southeast University, Nanjing 210096, China
}

Correspondence should be addressed to Yu-Ming Chu; chuyuming2005@yahoo.com.cn

Received 19 January 2013; Accepted 1 February 2013

Academic Editor: Khalil Ezzinbi

Copyright (C) 2013 Tie-Hong Zhao et al. This is an open access article distributed under the Creative Commons Attribution License, which permits unrestricted use, distribution, and reproduction in any medium, provided the original work is properly cited.

We prove that the double inequalities $I^{\alpha_{1}}(a, b) Q^{1-\alpha_{1}}(a, b)<M(a, b)<I^{\beta_{1}}(a, b) Q^{1-\beta_{1}}(a, b), I^{\alpha_{2}}(a, b) C^{1-\alpha_{2}}(a, b)<M(a, b)<$ $I^{\beta_{2}}(a, b) C^{1-\beta_{2}}(a, b)$ hold for all $a, b>0$ with $a \neq b$ if and only if $\alpha_{1} \geq 1 / 2, \beta_{1} \leq \log [\sqrt{2} \log (1+\sqrt{2})] /(1-\log \sqrt{2}), \alpha_{2} \geq 5 / 7$, and $\beta_{2} \leq \log [2 \log (1+\sqrt{2})]$, where $I(a, b), M(a, b), Q(a, b)$, and $C(a, b)$ are the identric, Neuman-Sándor, quadratic, and contraharmonic means of $a$ and $b$, respectively.

\section{Introduction}

For $p \in \mathbb{R}$ and $a, b>0$ with $a \neq b$, the identric mean $I(a, b)$, Neuman-Sándor mean $M(a, b)$ [1], quadratic mean $Q(a, b)$, contraharmonic mean $C(a, b)$, and $p$ th power mean $M_{p}(a, b)$ are defined by

$$
\begin{gathered}
I(a, b)=\frac{1}{e}\left(\frac{b^{b}}{a^{a}}\right)^{1 /(b-a)}, \\
M(a, b)=\frac{a-b}{2 \sinh ^{-1}[(a-b) /(a+b)]}, \\
Q(a, b)=\sqrt{\frac{a^{2}+b^{2}}{2},}, \quad C(a, b)=\frac{a^{2}+b^{2}}{a+b}, \\
M_{p}(a, b)= \begin{cases}\left(\frac{a^{p}+b^{p}}{2}\right)^{1 / p}, & p \neq 0, \\
\sqrt{a b}, & p=0,\end{cases}
\end{gathered}
$$

respectively, where $\sinh ^{-1}(x)=\log \left(x+\sqrt{1+x^{2}}\right)$ is the inverse hyperbolic sine function.
Recently, the identric, Neuman-Sándor, quadratic, and contraharmonic means have attracted the interest of numerous eminent mathematicians. In particular, many remarkable inequalities for these means can be found in the literature [1$18]$.

Let $H(a, b)=2 a b /(a+b), G(a, b)=\sqrt{a b}, L(a, b)=$ $(b-a) /(\log b-\log a), P(a, b)=(a-b) /(4 \arctan \sqrt{a / b}-\pi)$, $A(a, b)=(a+b) / 2$, and $T(a, b)=(a-b) /[2 \arctan ((a-b) /(a+$ $b)$ )] be the harmonic, geometric, logarithmic, first Seiffert, arithmetic, and second Seiffert means of two distinct positive numbers $a$ and $b$, respectively. Then it is well known that the inequalities

$$
\begin{aligned}
H(a, b) & =M_{-1}(a, b)<G(a, b)=M_{0}(a, b)<L(a, b) \\
& <P(a, b)<I(a, b)<A(a, b)<M_{1}(a, b) \\
& <M(a, b)<T(a, b)<Q(a, b)=M_{2}(a, b) \\
& <C(a, b)
\end{aligned}
$$


Neuman and Sándor $[1,8]$ established that

$$
\begin{gathered}
A(a, b)<M(a, b)<\frac{A(a, b)}{\log (1+\sqrt{2})}, \\
\frac{\pi}{4} T(a, b)<M(a, b)<T(a, b), \\
M(a, b)<\frac{A^{2}(a, b)}{P(a, b)}, \\
\sqrt{A(a, b) T(a, b)}<M(a, b) \\
<\sqrt{\frac{A^{2}(a, b)+T^{2}(a, b)}{2}} \\
M(a, b)<\frac{2 A(a, b)+Q(a, b)}{3}
\end{gathered}
$$

for all $a, b>0$ with $a \neq b$.

Let $0<a, b \leq 1 / 2$ with $a \neq b, a^{\prime}=1-a$, and $b^{\prime}=1-b$. Then the Ky Fan inequalities

$$
\begin{aligned}
\frac{G(a, b)}{G\left(a^{\prime}, b^{\prime}\right)} & <\frac{L(a, b)}{L\left(a^{\prime}, b^{\prime}\right)}<\frac{P(a, b)}{P\left(a^{\prime}, b^{\prime}\right)}<\frac{A(a, b)}{A\left(a^{\prime}, b^{\prime}\right)} \\
& <\frac{M(a, b)}{M\left(a^{\prime}, b^{\prime}\right)}<\frac{T(a, b)}{T\left(a^{\prime}, b^{\prime}\right)}
\end{aligned}
$$

were presented in [1].

$\mathrm{Li}$ et al. [19] found the best possible bounds for the Neuman-Sándor mean in terms of the generalized logarithmic mean $L_{r}(a, b)$. Neuman [20] and Zhao et al. [21] proved that the inequalities

$$
\begin{gathered}
\alpha Q(a, b)+(1-\alpha) A(a, b) \\
<M(a, b)<\beta Q(a, b)+(1-\beta) A(a, b), \\
\lambda C(a, b)+(1-\lambda) A(a, b) \\
<M(a, b)<\mu C(a, b)+(1-\mu) A(a, b), \\
\alpha_{1} H(a, b)+\left(1-\alpha_{1}\right) Q(a, b) \\
<M(a, b)<\beta_{1} H(a, b)+\left(1-\beta_{1}\right) Q(a, b), \\
\alpha_{2} C(a, b)+\left(1-\alpha_{2}\right) Q(a, b) \\
<M(a, b)<\beta_{2} C(a, b)+\left(1-\beta_{2}\right) Q(a, b)
\end{gathered}
$$

hold for all $a, b>0$ with $a \neq b$ if and only if $\alpha \leq[1-\log (1+$ $\sqrt{2})] /[(\sqrt{2}-1) \log (1+\sqrt{2})], \beta \geq 1 / 3, \lambda \leq[1-\log (1+$ $\sqrt{2})] / \log (1+\sqrt{2}), \mu \geq 1 / 6, \alpha_{1} \geq 2 / 9, \beta_{1} \leq 1-1 /[\sqrt{2} \log (1+$ $\sqrt{2})], \alpha_{2} \geq 1 / 3$, and $\beta_{2} \leq 1-1 /[\sqrt{2} \log (1+\sqrt{2})]$.

In [22], Chu and Long gave the best possible constants $p, q, \alpha$, and $\beta$ such that the double inequalities $M_{p}(a, b)<$ $M(a, b)<M_{q}(a, b)$ and $\alpha I(a, b)<M(a, b)<\beta I(a, b)$ hold for all $a, b>0$ with $a \neq b$.

The ratio of identric means leads to the weighted geometric mean

$$
\frac{I\left(a^{2}, b^{2}\right)}{I(a, b)}=\left(a^{a} b^{b}\right)^{1 /(a+b)}
$$

which has been investigated in [23-25]. Alzer [26] proved that the inequalities

$$
\begin{aligned}
\sqrt{A(a, b) G(a, b)} & <\sqrt{I(a, b) L(a, b)} \\
& <\frac{I(a, b)+L(a, b)}{2}<\frac{A(a, b)+G(a, b)}{2}
\end{aligned}
$$

hold for all $a, b>0$ with $a \neq b$.

The following sharp bounds for $I,(I L)^{1 / 2}$, and $(I+L) / 2$ in terms of the power mean and the convex combination of arithmetic and geometric means are given in [27] as

$$
\begin{aligned}
& M_{2 / 3}(a, b)<I(a, b)<M_{\log 2}(a, b) \\
& M_{0}(a, b)<\sqrt{I(a, b) L(a, b)}<M_{1 / 2}(a, b) \\
& M_{\log 2 /(1+\log 2)}(a, b) \\
& \quad<\frac{I(a, b)+L(a, b)}{2}<M_{1 / 2}(a, b) \\
& \frac{2}{3} A(a, b)+\frac{1}{3} G(a, b) \\
& \quad<I(a, b)<\frac{2}{e} A(a, b)+\left(1-\frac{2}{e}\right) G(a, b)
\end{aligned}
$$

for all $a, b>0$ with $a \neq b$.

Chu et al. [28] presented the optimal constants $\alpha_{1}, \beta_{1}, \alpha_{2}$, and $\beta_{2}$ such that the double inequalities

$$
\begin{aligned}
& \alpha_{1} Q(a, b)+\left(1-\alpha_{1}\right) A(a, b) \\
&<\frac{2}{\pi} \int_{0}^{\pi / 2} \sqrt{a^{2} \cos ^{2} \theta+b^{2} \sin ^{2} \theta} d \theta \\
&<\beta_{1} Q(a, b)+\left(1-\beta_{1}\right) A(a, b) \\
& Q^{\alpha_{2}}(a, b) A^{1-\alpha_{2}}(a, b) \\
&< \frac{2}{\pi} \int_{0}^{\pi / 2} \sqrt{a^{2} \cos ^{2} \theta+b^{2} \sin ^{2} \theta} d \theta \\
&<Q^{\beta_{2}}(a, b) A^{1-\beta_{2}}(a, b)
\end{aligned}
$$

hold for all $a, b>0$ with $a \neq b$.

The aim of this paper is to find the best possible constants $\alpha_{1}, \beta_{1}, \alpha_{2}$ and $\beta_{2}$ such that the double inequalities

$$
\begin{gathered}
I^{\alpha_{1}}(a, b) Q^{1-\alpha_{1}}(a, b)<M(a, b)<I^{\beta_{1}}(a, b) Q^{1-\beta_{1}}(a, b), \\
I^{\alpha_{2}}(a, b) C^{1-\alpha_{2}}(a, b)<M(a, b)<I^{\beta_{2}}(a, b) C^{1-\beta_{2}}(a, b)
\end{gathered}
$$

hold for all $a, b>0$ with $a \neq b$. All numerical computations are carried out using MATHEMATICA software.

\section{Lemmas}

In order to prove our main results, we need several lemmas, which we present in this section. 
Lemma 1. The double inequality

$$
x+\frac{x^{3}}{3}-\frac{2 x^{5}}{15}<\sqrt{1+x^{2}} \sinh ^{-1}(x)<x+\frac{x^{3}}{3}-\frac{2 x^{5}}{15}+\frac{8 x^{7}}{105}
$$

holds for $x \in(0,1)$.

Proof. To prove Lemma 1, it suffices to prove that

$$
\begin{gathered}
\eta_{1}(x)=\sqrt{1+x^{2}} \sinh ^{-1}(x)-\left(x+\frac{x^{3}}{3}-\frac{2 x^{5}}{15}\right)>0, \\
\eta_{2}(x)=\sqrt{1+x^{2}} \sinh ^{-1}(x)-\left(x+\frac{x^{3}}{3}-\frac{2 x^{5}}{15}+\frac{8 x^{7}}{105}\right)<0
\end{gathered}
$$

for $x \in(0,1)$.

From the expressions of $\eta_{1}(x)$ and $\eta_{2}(x)$, we get

$$
\begin{gathered}
\eta_{1}(0)=\eta_{2}(0)=0, \\
\eta_{1}^{\prime}(x)=\frac{x \eta_{1}^{*}(x)}{\sqrt{1+x^{2}}}, \quad \eta_{2}^{\prime}(x)=\frac{x \eta_{2}^{*}(x)}{\sqrt{1+x^{2}}}
\end{gathered}
$$

where

$$
\begin{gathered}
\eta_{1}^{*}(x)=\sinh ^{-1}(x)-\left(x-\frac{2 x^{3}}{3}\right) \sqrt{1+x^{2}}, \\
\eta_{2}^{*}(x)=\sinh ^{-1}(x)-\left(x-\frac{2 x^{3}}{3}+\frac{8 x^{5}}{15}\right) \sqrt{1+x^{2}}, \\
\eta_{1}^{*}(0)=\eta_{2}^{*}(0)=0 \\
\eta_{1}^{* \prime}(x)=\frac{8 x^{4}}{3 \sqrt{1+x^{2}}}>0, \\
\eta_{2}^{* \prime}(x)=-\frac{16 x^{6}}{5 \sqrt{1+x^{2}}}<0,
\end{gathered}
$$

for $x \in(0,1)$.

Therefore, inequality (12) follows from (14)-(16), and inequality (13) follows from (14)-(17).

Lemma 2. Let

$$
L(x)=\log \left[\frac{(1+x)^{1+x}}{(1-x)^{1-x}}\right]-2 x-x \log \left(1-x^{2}\right) .
$$

Then

$$
L(x)>\frac{2 x^{3}}{3}+\frac{2 x^{5}}{5}+\frac{2 x^{7}}{7}
$$

for $x \in(0,1)$, and

$$
L(x)<\frac{2 x^{3}}{3}+\frac{2 x^{5}}{5}+\frac{2 x^{7}}{7}+x^{9}
$$

for $x \in(0,3 / 4)$.
Proof. To prove inequalities (19) and (20), it suffices to show that

$$
\iota_{1}(x)
$$

$$
:=\log \left[\frac{(1+x)^{1+x}}{(1-x)^{1-x}}\right]-2 x-x \log \left(1-x^{2}\right)
$$

$$
-\left(\frac{2 x^{3}}{3}+\frac{2 x^{5}}{5}+\frac{2 x^{7}}{7}\right)>0
$$

for $x \in(0,1)$, and

$$
\iota_{2}(x)
$$

$$
\begin{aligned}
:= & \log \left[\frac{(1+x)^{1+x}}{(1-x)^{1-x}}\right]-2 x-x \log \left(1-x^{2}\right) \\
& -\left(\frac{2 x^{3}}{3}+\frac{2 x^{5}}{5}+\frac{2 x^{7}}{7}+x^{9}\right)<0
\end{aligned}
$$

for $x \in(0,3 / 4)$.

From (21) and (22), one has

$$
\begin{aligned}
& \iota_{1}\left(0^{+}\right)=\iota_{2}\left(0^{+}\right)=0, \\
& \iota_{1}^{\prime}(x)=\frac{2 x^{8}}{1-x^{2}}>0
\end{aligned}
$$

for $x \in(0,1)$, and

$$
\iota_{2}^{\prime}(x)=-\frac{9 x^{8}}{1-x^{2}}\left(\frac{7}{9}-x^{2}\right)<0
$$

for $x \in(0,3 / 4)$.

Therefore, inequality (21) follows from (23) and (24), and inequality (22) follows from (23) and (25).

Lemma 3. Let

$$
\Phi_{1}(x)=\frac{1}{\sqrt{1+x^{2}} \sinh ^{-1}(x)}-\frac{1}{x\left(1+x^{2}\right)} .
$$

Then the double inequality

$$
\frac{2 x}{3}-\frac{34 x^{3}}{45}+\frac{x^{5}}{2}<\Phi_{1}(x)<\frac{2 x}{3}-\frac{34 x^{3}}{45}+\frac{4 x^{5}}{5}
$$

holds for $x \in(0,0.7)$.

Proof. To prove inequality (27), it suffices to show that

$$
\begin{aligned}
\phi_{1}(x)= & x \sqrt{1+x^{2}}-\sinh ^{-1}(x) \\
& -x\left(1+x^{2}\right) \sinh ^{-1}(x)\left(\frac{2 x}{3}-\frac{34 x^{3}}{45}+\frac{x^{5}}{2}\right)
\end{aligned}
$$

$>0$,

$$
\begin{aligned}
\phi_{2}(x)= & x \sqrt{1+x^{2}}-\sinh ^{-1}(x) \\
& -x\left(1+x^{2}\right) \sinh ^{-1}(x)\left(\frac{2 x}{3}-\frac{34 x^{3}}{45}+\frac{4 x^{5}}{5}\right) \\
< & 0
\end{aligned}
$$

for $x \in(0,0.7)$. 
First, we prove inequality (28). From the expression of $\phi_{1}(x)$, we have

$$
\begin{gathered}
\phi_{1}(0)=0, \quad \phi_{1}(0.7)=0.0033 \cdots, \\
\phi_{1}^{\prime}(x)=\frac{x \phi_{1}^{*}(x)}{90 \sqrt{1+x^{2}}}
\end{gathered}
$$

where

$$
\begin{aligned}
\phi_{1}^{*}(x)= & 120 x+8 x^{3}+23 x^{5}-45 x^{7} \\
& -2\left(60-16 x^{2}-69 x^{4}+180 x^{6}\right) \\
& \times \sqrt{1+x^{2}} \sinh ^{-1}(x) .
\end{aligned}
$$

Equation (32) leads to

$$
\begin{gathered}
\phi_{1}^{*}(0.6)=3.017 \cdots, \quad \phi_{1}^{*}(0.7)=-3.551 \cdots, \\
\phi_{1}^{* \prime}(x)=-\frac{x \phi_{1}^{* *}(x)}{1+x^{2}},
\end{gathered}
$$

where

$$
\begin{aligned}
\phi_{1}^{* *}(x)= & -56 x-309 x^{3}+422 x^{5}+675 x^{7} \\
& +2\left(28-324 x^{2}+735 x^{4}+1260 x^{6}\right) \\
& \times \sqrt{1+x^{2}} \sinh ^{-1}(x) .
\end{aligned}
$$

Note that

$$
60-16 x^{2}-69 x^{4}+180 x^{6}>0
$$

for $x \in(0,0.6]$, and

$$
28-324 x^{2}+735 x^{4}+1260 x^{6}>0
$$

for $x \in[0.6,0.7)$. that

It follows from (32) and (34)-(36) together with Lemma 1

$$
\begin{aligned}
\phi_{1}^{*}(x)> & 120 x+8 x^{3}+23 x^{5}-45 x^{7} \\
& -2\left(60-16 x^{2}-69 x^{4}+180 x^{6}\right)\left(x+\frac{x^{3}}{3}\right) \\
& =\frac{x^{5}}{3}\left(515-1077 x^{2}-360 x^{4}\right) \\
\geq & \frac{x^{5}}{3}\left[515-1077 \times \frac{9}{25}-360 \times \frac{81}{625}\right] \\
= & \frac{10078 x^{5}}{375}>0
\end{aligned}
$$

for $x \in(0,0.6]$, and

$$
\begin{aligned}
\phi_{1}^{* *}(x)> & -56 x-309 x^{3}+422 x^{5}+675 x^{7} \\
& +2\left(28-324 x^{2}+735 x^{4}+1260 x^{6}\right) \\
& \times\left(x+\frac{x^{3}}{3}-\frac{2 x^{5}}{15}\right)=\frac{x^{3}}{15} \\
& \times\left(-14075+25028 x^{2}+56571 x^{4}+9660 x^{6}-5040 x^{8}\right) \\
> & \frac{x^{3}}{15}\left[-14075+25028 \times(0.6)^{2}+56571 \times(0.6)^{4}\right] \\
= & \frac{1416676 x^{3}}{9375}>0
\end{aligned}
$$

for $x \in[0.6,0.7)$.

From (33), (37), and (38), we clearly see that there exists $x_{1} \in(0.6,0.7)$ such that $\phi_{1}^{*}(x)>0$ for $x \in\left(0, x_{1}\right)$ and $\phi_{1}^{*}(x)<$ 0 for $x \in\left(x_{1}, 0.7\right)$. Then (31) leads to the conclusion that $\phi_{1}(x)$ is strictly increasing on $\left(0, x_{1}\right]$ and strictly decreasing on $\left[x_{1}, 0.7\right)$.

Therefore, inequality (28) follows from (30) and the piecewise monotonicity of $\phi_{1}(x)$.

Next, we prove inequality (29). From the expression of $\phi_{2}(x)$, we get

$$
\begin{gathered}
\phi_{2}(0)=0 \\
\phi_{2}^{\prime}(x)=-\frac{2 x \phi_{2}^{*}(x)}{45 \sqrt{1+x^{2}}}
\end{gathered}
$$

where

$$
\begin{aligned}
\phi_{2}^{*}(x)= & x\left(18 x^{6}+x^{4}-2 x^{2}-30\right) \\
& +2\left(15-4 x^{2}+3 x^{4}+72 x^{6}\right) \\
& \times \sqrt{1+x^{2}} \sinh ^{-1}(x)
\end{aligned}
$$

It follows from Lemma 1 and (40) that

$$
\begin{aligned}
\phi_{2}^{*}(x)> & x\left(18 x^{6}+x^{4}-2 x^{2}-30\right) \\
& +2\left(15-4 x^{2}+3 x^{4}+72 x^{6}\right)\left(x+\frac{x^{3}}{3}-\frac{2 x^{5}}{15}\right) \\
& =\frac{x^{5}}{15}\left(5+2476 x^{2}+708 x^{4}-288 x^{6}\right)>0
\end{aligned}
$$

for $x \in(0,0.7)$.

Therefore, inequality (29) follows from (39) together with (41).

Lemma 4. Let

$$
\Phi_{2}(x)=\frac{1}{\sqrt{1+x^{2}} \sinh ^{-1}(x)}-\frac{1-x^{2}}{x\left(1+x^{2}\right)} .
$$


Then the double inequality

$$
\frac{5 x}{3}-\frac{79 x^{3}}{45}+\frac{11 x^{5}}{10}<\Phi_{2}(x)<\frac{5 x}{3}-\frac{79 x^{3}}{45}+\frac{9 x^{5}}{5}
$$

holds for $x \in(0,3 / 4)$.

Proof. To prove Lemma 4, it suffices to prove that

$$
\begin{aligned}
\varphi_{1}(x):= & x \sqrt{1+x^{2}}-\left(1-x^{2}\right) \sinh ^{-1}(x) \\
& -x\left(1+x^{2}\right) \sinh ^{-1}(x)\left(\frac{5 x}{3}-\frac{79 x^{3}}{45}+\frac{11 x^{5}}{10}\right)>0,
\end{aligned}
$$$$
\varphi_{2}(x):=x \sqrt{1+x^{2}}-\left(1-x^{2}\right) \sinh ^{-1}(x)
$$$$
-x\left(1+x^{2}\right) \sinh ^{-1}(x)\left(\frac{5 x}{3}-\frac{79 x^{3}}{45}+\frac{9 x^{5}}{5}\right)<0
$$

for $x \in(0,3 / 4)$.

We first prove inequality (44). From the expression of $\varphi_{1}(x)$, we obtain

$$
\begin{gathered}
\varphi_{1}(0)=0, \quad \varphi_{1}\left(\frac{3}{4}\right)=0.008457 \cdots>0, \\
\varphi_{1}^{\prime}(x)=\frac{x \varphi_{1}^{*}(x)}{90 \sqrt{1+x^{2}}},
\end{gathered}
$$

where

$$
\begin{aligned}
\varphi_{1}^{*}(x)= & 120 x+8 x^{3}+59 x^{5}-99 x^{7}-2 \\
& \times\left(60-16 x^{2}-177 x^{4}+396 x^{6}\right) \sqrt{1+x^{2}} \sinh ^{-1}(x) .
\end{aligned}
$$

Equation (48) leads to

$$
\begin{gathered}
\varphi_{1}^{*}(0.66)=6.02 \cdots>0, \quad \varphi_{1}^{*}\left(\frac{3}{4}\right)=-19.299 \cdots<0 \\
\varphi_{1}^{* \prime}(x)=-\frac{x \phi_{1}^{* *}(x)}{1+x^{2}}
\end{gathered}
$$

where

$$
\begin{aligned}
\varphi_{1}^{* *}(x)= & -56 x-705 x^{3}+836 x^{5}+1485 x^{7} \\
& +14\left(4-108 x^{2}+213 x^{4}+396 x^{6}\right) \\
& \times \sqrt{1+x^{2}} \sinh ^{-1}(x) .
\end{aligned}
$$

Note that

$$
\begin{aligned}
60- & 16 x^{2}-177 x^{4}+396 x^{6} \\
> & 60-16 \times(0.66)^{2}-177 \times(0.66)^{4} \\
& =19.4451>0
\end{aligned}
$$

for $x \in(0,0.66)$, and

$$
\begin{aligned}
& 4-108 x^{2}+213 x^{4}+396 x^{6} \\
&> 4-108 \times\left(\frac{3}{4}\right)^{2}+213 \times(0.66)^{4} \\
&+396 \times(0.66)^{6}=16.3972>0
\end{aligned}
$$

for $x \in[0.66,3 / 4)$.

It follows from Lemma 1, (48), and (51)-(53) that

$$
\begin{aligned}
\varphi_{1}^{*}(x) & \\
> & 120 x+8 x^{3}+59 x^{5}-99 x^{7} \\
& -2\left(60-16 x^{2}-177 x^{4}+396 x^{6}\right) \\
& \times\left(x+\frac{x^{3}}{3}-\frac{2 x^{5}}{15}+\frac{8 x^{7}}{105}\right) \\
= & \frac{x^{5}}{105}\left[46165-82573 x^{2}-32420 x^{4}\right. \\
> & \frac{x^{5}}{105}\left[46165-82573 \times(0.66)^{2}-32420 \times(0.66)^{4}\right] \\
= & \frac{x^{5}}{105} \times 4044.5917 \cdots>0
\end{aligned}
$$

for $x \in(0,0.66)$, and

$\varphi_{1}^{* *}(x)>-56 x-705 x^{3}+836 x^{5}+1485 x^{7}$

$$
\begin{aligned}
& +14\left(4-108 x^{2}+213 x^{4}+396 x^{6}\right)\left(x+\frac{x^{3}}{3}-\frac{2 x^{5}}{15}\right) \\
= & \frac{x^{3}}{15}\left[-32975+49598 x^{2}+123369 x^{4}\right. \\
& \left.\quad+10668 x^{6}+11088 x^{6}\left(1-x^{2}\right)\right] \\
> & \frac{x^{3}}{15}\left[-32975+49598 \times(0.66)^{2}+123369 \times(0.66)^{4}\right] \\
= & \frac{x^{3}}{15} \times 12038.83 \cdots>0
\end{aligned}
$$

for $x \in[0.66,3 / 4)$.

From (50) and (55), we know that $\varphi_{1}^{*}(x)$ is strictly decreasing on $[0.66,3 / 4)$, and this in conjunction with (49) and (54) leads to the conclusion that there exists $x_{1} \in(0.66,3 / 4)$ such that $\varphi_{1}^{*}(x)>0$ for $x \in\left(0, x_{1}\right)$ and $\varphi_{1}^{*}(x)<0$ for $x \in\left(x_{1}, 3 / 4\right)$. Then (47) implies that $\varphi_{1}(x)$ is strictly increasing on $\left(0, x_{1}\right]$ and strictly decreasing on $\left[x_{1}, 3 / 4\right)$. Therefore, inequality (44) follows from (46) and the piecewise monotonicity of $\varphi_{1}(x)$. 
Next, we prove inequality (45). From the expression of $\varphi_{2}(x)$ one has

$$
\begin{gathered}
\varphi_{2}(0)=0 \\
\phi_{2}^{\prime}(x)=-\frac{x \phi_{2}^{*}(x)}{45 \sqrt{1+x^{2}}}
\end{gathered}
$$

where

$$
\begin{aligned}
\phi_{2}^{*}(x)= & -60 x-4 x^{3}+2 x^{5}+81 x^{7} \\
& +4\left(15-4 x^{2}+3 x^{4}+162 x^{6}\right) \\
& \times \sqrt{1+x^{2}} \sinh ^{-1}(x) .
\end{aligned}
$$

It follows from Lemma 1 and (52) that

$$
\begin{aligned}
\phi_{2}^{*}(x)> & -60 x-4 x^{3}+2 x^{5}+81 x^{7} \\
& +4\left(15-4 x^{2}+3 x^{4}+162 x^{6}\right)\left(x+\frac{x^{3}}{3}-\frac{2 x^{5}}{15}\right) \\
& =\frac{x^{5}}{15}\left(10+11027 x^{2}+3216 x^{4}-1296 x^{6}\right)>0
\end{aligned}
$$

for $x \in(0,3 / 4)$.

Therefore, inequality (45) follows from (56) together with (58).

Lemma 5. Let $L(x)$ be defined as in Lemma 2 and

$$
\Upsilon_{1}(x)=\frac{L(x)}{2 x^{2}}+\frac{x}{1+x^{2}}
$$

Then the double inequality

$$
\frac{4 x}{3}-\frac{4 x^{3}}{5}+\frac{4 x^{5}}{5}<\Upsilon_{1}(x)<\frac{4 x}{3}-\frac{4 x^{3}}{5}+\frac{8 x^{5}}{7}
$$

holds for $x \in(0,0.7)$.

Proof. From Lemma 2, one has

$$
\begin{aligned}
\Upsilon_{1}(x) & -\left(\frac{4 x}{3}-\frac{4 x^{3}}{5}+\frac{4 x^{5}}{5}\right) \\
> & \frac{1}{2 x^{2}}\left(\frac{2 x^{3}}{3}+\frac{2 x^{5}}{5}+\frac{2 x^{7}}{7}\right)+\frac{x}{1+x^{2}} \\
& -\left(\frac{4 x}{3}-\frac{4 x^{3}}{5}+\frac{4 x^{5}}{5}\right)
\end{aligned}
$$

$$
\begin{aligned}
= & \frac{23 x^{5}}{35\left(1+x^{2}\right)}\left(\frac{12}{23}-x^{2}\right)>0 \\
\Upsilon_{1}(x) & -\left(\frac{4 x}{3}-\frac{4 x^{3}}{5}+\frac{8 x^{5}}{7}\right) \\
< & \frac{1}{2 x^{2}}\left(\frac{2 x^{3}}{3}+\frac{2 x^{5}}{5}+\frac{2 x^{7}}{7}+x^{9}\right) \\
& +\frac{x}{1+x^{2}}-\left(\frac{4 x}{3}-\frac{4 x^{3}}{5}+\frac{8 x^{5}}{7}\right) \\
= & -\frac{x^{7}\left(1-x^{2}\right)}{2\left(1+x^{2}\right)}<0
\end{aligned}
$$

for $x \in(0,0.7)$.

Therefore, Lemma 5 follows easily from (61).

Lemma 6. Let $L(x)$ be defined as in Lemma 2 and

$$
\Upsilon_{2}(x)=\frac{L(x)}{2 x^{2}}+\frac{2 x}{1+x^{2}} .
$$

Then the double inequality

$$
\frac{7 x}{3}-\frac{9 x^{3}}{5}+\frac{7 x^{5}}{5}<\Upsilon_{2}(x)<\frac{7 x}{3}-\frac{9 x^{3}}{5}+\frac{15 x^{5}}{7}
$$

holds for $x \in(0,3 / 4)$.

Proof. It follows from Lemma 2 that

$$
\begin{aligned}
\Upsilon_{2}(x) & -\left(\frac{7 x}{3}-\frac{9 x^{3}}{5}+\frac{7 x^{5}}{5}\right) \\
> & \frac{1}{2 x^{2}}\left(\frac{2 x^{3}}{3}+\frac{2 x^{5}}{5}+\frac{2 x^{7}}{7}\right) \\
& +\frac{2 x}{1+x^{2}}-\left(\frac{7 x}{3}-\frac{9 x^{3}}{5}+\frac{7 x^{5}}{5}\right) \\
= & \frac{44 x^{5}}{35\left(1+x^{2}\right)}\left(\frac{13}{22}-x^{2}\right)>0, \\
\Upsilon_{2}(x) & -\left(\frac{7 x}{3}-\frac{9 x^{3}}{5}+\frac{15 x^{5}}{7}\right) \\
< & \frac{1}{2 x^{2}}\left(\frac{2 x^{3}}{3}+\frac{2 x^{5}}{5}+\frac{2 x^{7}}{7}+x^{9}\right) \\
& +\frac{2 x}{1+x^{2}}-\left(\frac{7 x}{3}-\frac{9 x^{3}}{5}+\frac{15 x^{5}}{7}\right) \\
= & \frac{x^{7}\left(3-x^{2}\right)}{2\left(1+x^{2}\right)}<0
\end{aligned}
$$

for $x \in(0,3 / 4)$.

Therefore, Lemma 6 follows from (64). 
Lemma 7. The inequality

$$
\frac{x^{3}}{\sqrt{1+x^{2}}}>\left[\sinh ^{-1}(x)\right]^{3}
$$

holds for $x \in(0,1)$.

Proof. Let

$$
\zeta(x)=\frac{x^{3}}{\sqrt{1+x^{2}}}-\left[\sinh ^{-1}(x)\right]^{3}
$$

Then

$$
\begin{gathered}
\zeta(0)=0, \\
\zeta^{\prime}(x)=\frac{\zeta_{1}(x)}{\left(1+x^{2}\right)^{3 / 2}},
\end{gathered}
$$

where

$$
\zeta_{1}(x)=x^{2}\left(3+2 x^{2}\right)-3\left[\sqrt{1+x^{2}} \sinh ^{-1}(x)\right]^{2} .
$$

It follows from Lemma 1 and (68) that

$\zeta_{1}(x)$

$$
\begin{gathered}
>x^{2}\left(3+2 x^{2}\right)-3\left(x+\frac{x^{3}}{3}-\frac{2 x^{5}}{15}+\frac{8 x^{7}}{105}\right)^{2} \\
=x^{6}\left[\frac{37}{525}+\left(\frac{208}{525}+\frac{36 x^{2}}{175}+\frac{64 x^{6}}{3675}\right)\right. \\
\left.\times\left(1-x^{2}\right)+\frac{32 x^{6}}{735}\right]>0
\end{gathered}
$$

for $x \in(0,1)$.

Therefore, Lemma 7 follows from (67) together with (69).

Lemma 8. Let

$$
\begin{aligned}
\mu_{1}(x)= & \frac{1+3 x^{2}}{\left(x+x^{3}\right)^{2}}-\frac{1}{\left(1+x^{2}\right)\left[\sinh ^{-1}(x)\right]^{2}} \\
& -\frac{x}{\left(1+x^{2}\right)^{3 / 2} \sinh ^{-1}(x)} .
\end{aligned}
$$

Then $\mu_{1}(x)<0.2$ for $x \in[0.7,1)$.

Proof. Let

$$
\begin{aligned}
& \omega_{1}(x)=\frac{1}{x^{2}}-\frac{1}{\left[\sinh ^{-1}(x)\right]^{2}}, \\
& \omega_{2}(x)=\frac{2}{\sqrt{1+x^{2}}}-\frac{x}{\sinh ^{-1}(x)} .
\end{aligned}
$$

Then

$$
\mu_{1}(x)=\frac{\omega_{1}(x)}{1+x^{2}}+\frac{\omega_{2}(x)}{\left(1+x^{2}\right)^{3 / 2}}
$$

Lemma 7 and $x>\sinh ^{-1}(x)$ give $\omega_{1}(x)<0$ and

$$
\omega_{1}^{\prime}(x)=\frac{2}{x^{3}\left[\sinh ^{-1}(x)\right]^{3}}\left[\frac{x^{3}}{\sqrt{1+x^{2}}}-\left(\sinh ^{-1}(x)\right)^{3}\right]>0
$$

for $x \in(0,1)$. This in turn implies that

$$
\left[\frac{\omega_{1}(x)}{1+x^{2}}\right]^{\prime}=\frac{\omega_{1}^{\prime}(x)\left(1+x^{2}\right)-2 x \omega_{1}(x)}{\left(1+x^{2}\right)^{2}}>0
$$

for $x \in(0,1)$.

On the other hand, from the expression of $\omega_{2}(x)$, we get

$$
\begin{gathered}
\omega_{2}(1)=0.2796 \cdots>0, \\
\omega_{2}^{\prime}(x)=-\frac{2 x}{\left(1+x^{2}\right)^{3 / 2}}+\frac{\omega_{2}^{*}(x)}{\left[\sinh ^{-1}(x)\right]^{2}},
\end{gathered}
$$

where

$$
\begin{gathered}
\omega_{2}^{*}(x)=\frac{x}{\sqrt{1+x^{2}}}-\sinh ^{-1}(x), \\
\omega_{2}^{*}(0)=0, \\
\omega_{2}^{* \prime}(x)=-\frac{x^{2}}{\left(1+x^{2}\right)^{3 / 2}}<0
\end{gathered}
$$

for $x \in(0,1)$.

From (75)-(76), we clearly see that $\omega_{2}^{\prime}(x)<0$ and $\omega_{2}(x)>$ 0 for $x \in(0,1)$. This in turn implies that

$$
\begin{aligned}
& {\left[\frac{\omega_{2}(x)}{\left(1+x^{2}\right)^{3 / 2}}\right]^{\prime}} \\
& \quad=\frac{\omega_{2}^{\prime}(x)\left(1+x^{2}\right)^{3 / 2}-3 x \sqrt{1+x^{2}} \omega_{2}(x)}{\left(1+x^{2}\right)^{3}}
\end{aligned}
$$$$
<0
$$

for $x \in(0,1)$.

Equation (72) together with inequalities (74) and (77) lead to the conclusion that

$$
\begin{aligned}
& \mu_{1}(x) \\
& \leq \frac{\omega_{1}(1)}{2}+\frac{\omega_{2}(0.7)}{\left[1+(0.7)^{2}\right]^{3 / 2}} \\
& \quad=0.167 \cdots<0.2
\end{aligned}
$$

for $x \in[0.7,1)$.

Lemma 9. Let

$$
\begin{aligned}
\mu_{2}(x)= & \frac{1+4 x^{2}-x^{4}}{\left(x+x^{3}\right)^{2}}-\frac{1}{\left(1+x^{2}\right)\left[\sinh ^{-1}(x)\right]^{2}} \\
& -\frac{x}{\left(1+x^{2}\right)^{3 / 2} \sinh ^{-1}(x)} .
\end{aligned}
$$

Then $\mu_{2}(x)<0.51$ for $x \in[0.65,1)$. 
Proof. Let

$$
\begin{aligned}
& \tau_{1}(x)=\frac{1}{x^{2}}-\frac{1}{\left[\sinh ^{-1}(x)\right]^{2}}=\mu_{1}(x), \\
& \tau_{2}(x)=\frac{3-x^{2}}{\sqrt{1+x^{2}}}-\frac{x}{\sinh ^{-1}(x)},
\end{aligned}
$$

then

$$
\mu_{2}(x)=\frac{\tau_{1}(x)}{1+x^{2}}+\frac{\tau_{2}(x)}{\left(1+x^{2}\right)^{3 / 2}} .
$$

From (74), we clearly see that

$$
\left[\frac{\tau_{1}(x)}{1+x^{2}}\right]^{\prime}=\left[\frac{\omega_{1}(x)}{1+x^{2}}\right]^{\prime}>0
$$

for $x \in(0,1)$.

On the other hand, from the expression of $\tau_{2}(x)$ together with Lemma 1, we get

$$
\begin{gathered}
\tau_{2}(1)=0.2796 \cdots>0, \\
\tau_{2}^{\prime}(x)=-\frac{1}{\sinh ^{-1}(x)}-\frac{x \tau_{2}^{*}(x)}{\left(1+x^{2}\right)^{3 / 2}\left[\sinh ^{-1}(x)\right]^{2}}, \\
\tau_{2}^{*}(x)=\left(5+x^{2}\right)\left[\sinh ^{-1}(x)\right]^{2}-\left(1+x^{2}\right), \\
\tau_{2}^{*}(0.65)=0.6033 \cdots, \\
\tau_{2}^{* \prime}(x)=2 x\left[\sinh ^{-1}(x)\right]^{2} \\
+2\left[\frac{5+x^{2}}{1+x^{2}} \sqrt{1+x^{2}} \sinh ^{-1}(x)-x\right]>0
\end{gathered}
$$

for $x \in(0,1)$.

From (83), we clearly see that $\tau_{2}^{\prime}(x)<0$ and $\tau_{2}(x)>0$ for $x \in[0.65,1)$. This in turn implies that

$$
\left[\frac{\tau_{2}(x)}{\left(1+x^{2}\right)^{3 / 2}}\right]^{\prime}=\frac{\tau_{2}^{\prime}(x)\left(1+x^{2}\right)^{3 / 2}-3 x \sqrt{1+x^{2}} \tau_{2}(x)}{\left(1+x^{2}\right)^{3}}<0
$$

for $x \in[0.65,1)$.

Equation (81) together with inequalities (82) and (84) lead to the conclusion that

$$
\mu_{2}(x) \leq \frac{\tau_{1}(1)}{2}+\frac{\tau_{2}(0.65)}{\left[1+(0.65)^{2}\right]^{3 / 2}}=0.503 \cdots<0.51
$$

for $x \in[0.65,1)$.

Lemma 10. Let $L(x)$ be defined as in Lemma 2 and

$$
\nu_{1}(x)=\frac{2\left(1+x^{4}\right)}{\left(1-x^{2}\right)\left(1+x^{2}\right)^{2}}-\frac{L(x)}{x^{3}} .
$$

Then $v_{1}(x)>1.2$ for $x \in[0.7,1)$.
Proof. Differentiating $\nu_{1}(x)$ yields

$$
\nu_{1}^{\prime}(x)=\frac{3 L(x)}{x^{4}}-\frac{2+8 x^{2}-20 x^{4}-6 x^{8}}{x\left(1-x^{2}\right)^{2}\left(1+x^{2}\right)^{3}} .
$$

It follows from (19) and (87) that

$v_{1}^{\prime}(x)$

$$
\begin{aligned}
& >\frac{1}{x}\left[3\left(\frac{2}{3}+\frac{2 x^{2}}{5}+\frac{2 x^{4}}{7}\right)-\frac{2+8 x^{2}-20 x^{4}-6 x^{8}}{\left(1-x^{2}\right)^{2}\left(1+x^{2}\right)^{3}}\right] \\
& =\frac{2 x\left(-84+316 x^{2}-97 x^{4}+68 x^{6}+26 x^{8}+36 x^{10}+15 x^{12}\right)}{35\left(1-x^{2}\right)^{2}\left(1+x^{2}\right)^{3}} \\
& >\frac{2 x}{35\left(1-x^{2}\right)^{2}\left(1+x^{2}\right)^{3}}\left[-84+316 \times(0.7)^{2}-\frac{349}{5}\right. \\
& >\frac{2 x}{35\left(1-x^{2}\right)^{2}\left(1+x^{2}\right)^{3}}>0
\end{aligned}
$$

for $x \in[0.7,1)$.

Therefore, $\nu_{1}(x) \geq \nu_{1}(0.7)=1.214 \cdots>1.2$ for $x \in$ $[0.7,1)$ follows from $(88)$.

Lemma 11. Let $L(x)$ be defined as in Lemma 2 and

$$
\nu_{2}(x)=\frac{3-2 x^{2}+3 x^{4}}{\left(1-x^{2}\right)\left(1+x^{2}\right)^{2}}-\frac{L(x)}{x^{3}} .
$$

Then $\nu_{2}(x)>1.38$ for $x \in[0.65,1)$.

Proof. Differentiating $v_{2}(x)$ yields

$$
v_{2}^{\prime}(x)=\frac{3 L(x)}{x^{4}}-\frac{2\left(1+7 x^{2}-17 x^{4}+5 x^{6}-4 x^{8}\right)}{x\left(1-x^{2}\right)^{2}\left(1+x^{2}\right)^{3}} .
$$

It follows from (19) and (90) together with the monotonicity of the function $561 x^{2}-272 x^{4}$ on $[0.65,1)$ that

$$
\begin{aligned}
v_{2}^{\prime}(x) & \\
& >\frac{1}{x}\left[3\left(\frac{2}{3}+\frac{2 x^{2}}{5}+\frac{2 x^{4}}{7}\right)-\frac{2\left(1+7 x^{2}-17 x^{4}+5 x^{6}-4 x^{8}\right)}{\left(1-x^{2}\right)^{2}\left(1+x^{2}\right)^{3}}\right] \\
& =\frac{2 x\left(-189+561 x^{2}-272 x^{4}+103 x^{6}+26 x^{8}+36 x^{10}+15 x^{12}\right)}{35\left(1-x^{2}\right)^{2}\left(1+x^{2}\right)^{3}} \\
& >\frac{2 x\left[-189+561 \times(0.65)^{2}-272 \times(0.65)^{4}+103 \times(0.65)^{6}\right]}{35\left(1-x^{2}\right)^{2}\left(1+x^{2}\right)^{3}} \\
& =\frac{2 x \times 7.23 \cdots}{35\left(1-x^{2}\right)^{2}\left(1+x^{2}\right)^{3}}>0
\end{aligned}
$$

for $x \in[0.65,1)$.

Equation (91) leads to the conclusion that $v_{2}(x) \geq$ $v_{2}(0.65)=1.389 \cdots>1.38$ for $x \in[0.65,1)$. 
Lemma 12. Let $\Phi_{1}(x)$ and $\Upsilon_{1}(x)$ be defined, respectively, as in Lemmas 3 and 5 , and $\Theta_{1}(x ; p)=\Phi_{1}(x)-p \Upsilon_{1}(x)$. Then $\Theta_{1}(x ; p)$ is strictly decreasing on $[0.7,1)$ if $p>1 / 6$.

Proof. Differentiating $\Theta_{1}(x ; p)$ with respect to $x$ and making use of Lemmas 8 and 10, we get

$$
\begin{aligned}
\frac{d \Theta_{1}(x ; p)}{d x} & =\Phi_{1}^{\prime}(x)-p \Upsilon_{1}^{\prime}(x)=\mu_{1}(x)-p \nu_{1}(x) \\
& <0.2-\frac{1}{6} \times 1.2=0
\end{aligned}
$$

for $x \in[0.7,1)$ and $p>1 / 6$. This in turn implies that $\Theta_{1}(x ; p)$ is strictly decreasing on $[0.7,1)$ if $p>1 / 6$.

Lemma 13. Let $\Phi_{2}(x)$ and $\Upsilon_{2}(x)$ be defined, respectively, as in Lemmas 4 and 6 , and $\Theta_{2}(x ; q)=\Phi_{2}(x)-q \Upsilon_{2}(x)$. Then $\Theta_{2}(x ; q)$ is strictly decreasing on $[0.65,1)$ if $q>2 / 5$.

Proof. Differentiating $\Theta_{2}(x ; q)$ with respect to $x$ and making use of Lemmas 9 and 11, we have

$$
\begin{aligned}
\frac{d \Theta_{1}(x ; q)}{d x} & =\Phi_{2}^{\prime}(x)-q \Upsilon_{2}^{\prime}(x)=\mu_{2}(x)-q \nu_{2}(x) \\
& <0.51-\frac{2}{5} \times 1.38=-0.042<0
\end{aligned}
$$

for $x \in[0.65,1)$ and $q>2 / 5$. This in turn implies that $\Theta_{2}(x ; q)$ is strictly decreasing on $[0.65,1)$ if $q>2 / 5$.

\section{Main Results}

Theorem 14. The double inequality

$$
I^{\alpha_{1}}(a, b) Q^{1-\alpha_{1}}(a, b)<M(a, b)<I^{\beta_{1}}(a, b) Q^{1-\beta_{1}}(a, b)
$$

holds for all $a, b>0$ with $a \neq b$ if and only if $\beta_{1} \leq$ $\log [\sqrt{2} \log (1+\sqrt{2})] /(1-\log \sqrt{2})=0.337 \cdots$ and $\alpha_{1} \geq 1 / 2$.

Proof. Since $I(a, b), M(a, b)$, and $Q(a, b)$ are symmetric and homogeneous of degree one, then without loss of generality, we assume that $a>b$. Let $p \in(0,1), x=(a-b) /(a+b)$, and $\lambda_{1}=\log [\sqrt{2} \log (1+\sqrt{2})] /(1-\log \sqrt{2})$. Then $x \in(0,1)$, and

$$
\begin{aligned}
& \frac{I(a, b)}{A(a, b)}=\frac{1}{e}\left[\frac{(1+x)^{1+x}}{(1-x)^{1-x}}\right]^{1 / 2 x}, \\
& \frac{M(a, b)}{A(a, b)}=\frac{x}{\sinh ^{-1}(x)}, \quad \frac{Q(a, b)}{A(a, b)}=\sqrt{1+x^{2}}, \\
& \frac{\log [Q(a, b)]-\log [M(a, b)]}{\log [Q(a, b)]-\log [I(a, b)]} \\
& \quad=\frac{\log \sqrt{1+x^{2}}-\log x+\log \left[\sinh ^{-1}(x)\right]}{\log \sqrt{1+x^{2}}-\log \left[(1+x)^{1+x} /(1-x)^{1-x}\right] /(2 x)+1},
\end{aligned}
$$

$$
\begin{aligned}
\lim _{x \rightarrow 0^{+}} & \frac{\log \sqrt{1+x^{2}}-\log x+\log \left[\sinh ^{-1}(x)\right]}{\sqrt{1+x^{2}}-\log \left[(1+x)^{1+x} /(1-x)^{1-x}\right] /(2 x)+1} \\
& =\frac{1}{2},
\end{aligned}
$$

$$
\begin{aligned}
\lim _{x \rightarrow 1^{-}} & \frac{\log \sqrt{1+x^{2}}-\log x+\log \left[\sinh ^{-1}(x)\right]}{\log \sqrt{1+x^{2}}-\log \left[(1+x)^{1+x} /(1-x)^{1-x}\right] /(2 x)+1} \\
& =\lambda_{1} .
\end{aligned}
$$

The difference between the convex combination of $\log [I(a, b)], \log [Q(a, b)]$ and $\log [M(a, b)]$ is as follows:

$$
\begin{aligned}
p \log [ & I(a, b)]+(1-p) \log [Q(a, b)]-\log [M(a, b)] \\
= & \frac{p}{2 x} \log \left[\frac{(1+x)^{1+x}}{(1-x)^{1-x}}\right]-p \\
& +(1-p) \log \sqrt{1+x^{2}}-\log \left[\frac{x}{\sinh ^{-1}(x)}\right]:=D_{p}(x) .
\end{aligned}
$$

Equation (99) leads to

$$
\begin{gathered}
D_{p}\left(0^{+}\right)=0 \\
D_{p}\left(1^{-}\right)=\log [\sqrt{2} \log (1+\sqrt{2})]-p(1-\log \sqrt{2}) \\
D_{\lambda_{1}}\left(1^{-}\right)=0 \\
D_{p}^{\prime}(x)=-\frac{1+p x^{2}}{x+x^{3}}+\frac{1}{\sqrt{1+x^{2}} \sinh ^{-1}(x)}-\frac{L(x)}{2 x^{2}} \\
=\Phi_{1}(x)-p \Upsilon_{1}(x)=\Theta_{1}(x ; p)
\end{gathered}
$$

where $L(x), \Phi_{1}(x), \Upsilon_{1}(x)$, and $\Theta_{1}(x ; p)$ are defined as in Lemmas 2, 3, 5, and 12, respectively. 
It follows from (101) together with Lemmas 3 and 5 that $D_{1 / 2}^{\prime}(x)$

$$
\begin{aligned}
& <\frac{2 x}{3}-\frac{34 x^{3}}{45}+\frac{4 x^{5}}{5}-\frac{1}{2}\left(\frac{4 x}{3}-\frac{4 x^{3}}{5}+\frac{4 x^{5}}{5}\right) \\
& =-\frac{2 x^{2}}{5}\left(\frac{8}{9}-x^{2}\right)<0
\end{aligned}
$$

for $x \in(0,0.7)$. Moreover, we see clearly, from Lemma 12, that $D_{1 / 2}^{\prime}(x)$ is strictly decreasing on $[0.7,1)$ and so $D_{1 / 2}^{\prime}(x)<$ $D_{1 / 2}^{\prime}(0.7)=-0.109 \cdots<0$ for $x \in[0.7,1)$. This in conjunction with (100) and (102) implies that

$$
D_{1 / 2}(x)<0
$$

for $x \in(0,1)$.

On the other hand, (101) and Lemmas 3 and 5 together with the monotonicity of the function $-2\left(17-18 \lambda_{1}\right) x^{2} / 45+$ $\left(7-16 \lambda_{1}\right) x^{4} / 14$ on $(0,0.7)$ lead to

$$
\begin{aligned}
D_{\lambda_{1}}^{\prime}(x) & \\
> & \frac{2 x}{3}-\frac{34 x^{3}}{45}+\frac{x^{5}}{2}-\lambda_{1}\left(\frac{4 x}{3}-\frac{4 x^{3}}{5}+\frac{8 x^{5}}{7}\right) \\
& =x\left[\frac{2\left(1-2 \lambda_{1}\right)}{3}-\frac{2\left(17-18 \lambda_{1}\right)}{45} x^{2}+\frac{7-16 \lambda_{1}}{14} x^{4}\right] \\
> & x\left[\frac{2\left(1-2 \lambda_{1}\right)}{3}-\frac{2\left(17-18 \lambda_{1}\right)}{45} \times(0.7)^{2}\right. \\
& \left.+\frac{7-16 \lambda_{1}}{14} \times(0.7)^{4}\right] \\
= & \frac{\left(74969-218832 \lambda_{1}\right) x}{180000}>0
\end{aligned}
$$

for $x \in(0,0.7)$.

It follows from Lemma 12 that $D_{\lambda_{1}}^{\prime}(x)$ is strictly decreasing on $[0.7,1)$. Note that

$$
D_{\lambda_{1}}^{\prime}(0.7)=0.0229 \cdots>0, \quad D_{\lambda_{1}}^{\prime}\left(1^{-}\right)=-\infty .
$$

From (104) and (105) together with the monotonicity of $D_{\lambda_{1}}^{\prime}(x)$ on $[0.7,1)$, we clearly see that there exists $c_{1} \in(0.7,1)$ such that $D_{\lambda_{1}}(x)$ is strictly increasing on $\left(0, c_{1}\right]$ and strictly decreasing on $\left[c_{1}, 1\right)$. This in conjunction with (100) implies that

$$
D_{\lambda_{1}}(x)>0
$$

for $x \in(0,1)$.

Equation (99) together with inequalities (103) and (106) gives rise to

$$
\begin{aligned}
& M(a, b)>I^{1 / 2}(a, b) Q^{1 / 2}(a, b), \\
& M(a, b)<I^{\lambda_{1}}(a, b) Q^{1-\lambda_{1}}(a, b) .
\end{aligned}
$$

Therefore, Theorem 14 follows from (107) together with the following statements. (i) If $\alpha_{1}<1 / 2$, then (96) and (97) imply that there exists $\delta_{1} \in(0,1)$ such that $M(a, b)<I^{\alpha_{1}}(a, b) Q^{1-\alpha_{1}}(a, b)$ for all $a, b>0$ with $(a-b) /(a+b) \in\left(0, \delta_{1}\right)$.

(ii) If $\beta_{1}>\lambda_{1}$, then (96) and (98) imply that there exists $\delta_{2} \in(0,1)$ such that $M(a, b)>I^{\beta_{1}}(a, b) Q^{1-\beta_{1}}(a, b)$ for all $a, b>0$ with $(a-\mathrm{b}) /(a+b) \in\left(1-\delta_{2}, 1\right)$.

Theorem 15. The double inequality

$$
I^{\alpha_{2}}(a, b) C^{1-\alpha_{2}}(a, b)<M(a, b)<I^{\beta_{2}}(a, b) C^{1-\beta_{2}}(a, b)
$$

holds for all $a, b>0$ with $a \neq b$ if and only if $\alpha_{2} \geq 5 / 7$ and $\beta_{2} \leq \log [2 \log (1+\sqrt{2})]=0.566 \cdots$.

Proof. We will follow the same idea in the proof of Theorem 14 . Since $I(a, b), M(a, b)$, and $C(a, b)$ are symmetric and homogeneous of degree one. Without loss of generality, we assume that $a>b$. Let $q \in(0,1), \lambda_{2}=\log [2 \log (1+\sqrt{2})]$, and $x=(a-b) /(a+b)$. Then $x \in(0,1)$.

Making use of (95) together with $C(a, b) / A(a, b)=1+x^{2}$ gives

$$
\begin{aligned}
& \frac{\log [C(a, b)]-\log [M(a, b)]}{\log [C(a, b)]-\log [I(a, b)]} \\
& \quad=\frac{\log \left(1+x^{2}\right)-\log x+\log \left[\sinh ^{-1}(x)\right]}{\log \left(1+x^{2}\right)-\log \left[(1+x)^{1+x} /(1-x)^{1-x}\right] /(2 x)+1}, \\
& \lim _{x \rightarrow 0^{+}} \frac{\log \left(1+x^{2}\right)-\log x+\log \left[\sinh ^{-1}(x)\right]}{\log \left(1+x^{2}\right)-\log \left[(1+x)^{1+x} /(1-x)^{1-x}\right] /(2 x)+1} \\
& =\frac{5}{7},
\end{aligned}
$$

$$
\begin{aligned}
\lim _{x \rightarrow 1^{-}} & \frac{\log \left(1+x^{2}\right)-\log x+\log \left[\sinh ^{-1}(x)\right]}{\log \left(1+x^{2}\right)-\log \left[(1+x)^{1+x} /(1-x)^{1-x}\right] /(2 x)+1} \\
& =\lambda_{2} .
\end{aligned}
$$

The difference between the convex combination of $\log [I(a, b)], \log [C(a, b)]$ and $\log [M(a, b)]$ is as follows:

$$
\begin{aligned}
q \log [ & {[(a, b)]+(1-q) \log [C(a, b)]-\log [M(a, b)] } \\
= & \frac{q}{2 x} \log \left[\frac{(1+x)^{1+x}}{(1-x)^{1-x}}\right]-q+(1-q) \log \left(1+x^{2}\right) \\
& -\log \left[\frac{x}{\sinh ^{-1}(x)}\right]:=E_{q}(x) .
\end{aligned}
$$


Equation (112) leads to

$$
\begin{aligned}
& E_{q}\left(0^{+}\right)=0, \quad E_{q}\left(1^{-}\right)=\log [2 \log (1+\sqrt{2})]-q, \\
& E_{\lambda_{2}}\left(1^{-}\right)=0, \\
& E_{q}^{\prime}(x) \\
& \quad=-\frac{1-x^{2}+2 q x^{2}}{x+x^{3}}+\frac{1}{\sqrt{1+x^{2}} \sinh ^{-1}(x)}-\frac{L(x)}{2 x^{2}} \\
& \quad=\Phi_{2}(x)-q \Upsilon_{2}(x)=\Theta_{2}(x ; q),
\end{aligned}
$$

where $L(x), \Phi_{2}(x), \Upsilon_{2}(x)$, and $\Theta_{2}(x ; q)$ are defined as in Lemmas $2,4,6$, and 13 , respectively. that

It follows from Lemmas 4, 6, and 13 together with (114)

$$
\begin{aligned}
& E_{5 / 7}^{\prime}(x) \\
& <\left(\frac{5 x}{3}-\frac{79 x^{3}}{45}+\frac{9 x^{5}}{5}\right)-\frac{5}{7}\left(\frac{7 x}{3}-\frac{9 x^{3}}{5}+\frac{7 x^{5}}{5}\right) \\
& =-\frac{4 x^{2}}{5}\left(\frac{37}{63}-x^{2}\right)<0
\end{aligned}
$$

for $x \in(0,0.65)$ and $E_{5 / 7}^{\prime}(x)$ is strictly decreasing on $[0.65,1)$. Thus, we have $E_{5 / 7}^{\prime}(x)<E_{5 / 7}^{\prime}(0.65)=-0.117 \cdots$ for $x \in$ $[0.65,1)$. This in conjunction with (113) and (115) implies that

$$
E_{5 / 7}(x)<0
$$

for $x \in(0,1)$.

On the other hand, Lemmas 4, 6, and 13 together with (114) lead to

$$
\begin{aligned}
& E_{\lambda_{2}}^{\prime}(x) \\
& >\left(\frac{5 x}{3}-\frac{79 x^{3}}{45}+\frac{11 x^{5}}{10}\right)-\lambda_{2}\left(\frac{7 x}{3}-\frac{9 x^{3}}{5}+\frac{15 x^{5}}{7}\right) \\
& =x\left[\frac{5-7 \lambda_{2}}{3}-\frac{79-81 \lambda_{2}}{45} x^{2}-\frac{150 \lambda_{2}-77}{70} x^{4}\right] \\
& >x\left[\frac{5-7 \lambda_{2}}{3}-\frac{79-81 \lambda_{2}}{45} \times(0.65)^{2}\right. \\
& \left.=\frac{150 \lambda_{2}-77}{70} \times(0.65)^{4}\right] \\
& 100800000
\end{aligned}
$$

for $x \in(0,0.65)$ and $E_{\lambda_{2}}^{\prime}(x)$ is strictly decreasing on $[0.65,1)$. Note that

$$
E_{\lambda_{2}}^{\prime}(0.65)=0.0609 \cdots, \quad E_{\lambda_{2}}^{\prime}\left(1^{-}\right)=-\infty .
$$

From (117) and (118) together with the monotonicity of $E_{\lambda_{2}}^{\prime}(x)$ on $[0.65,1)$, we clearly see that there exists $c_{2} \in(0.65,1)$ such that $E_{\lambda_{2}}(x)$ is strictly increasing on $\left(0, c_{2}\right]$ and strictly decreasing on $\left[c_{2}, 1\right)$. This in conjunction with (113) implies that

$$
E_{\lambda_{2}}(x)>0
$$

for $x \in(0,1)$.

Equation (112) together with inequalities (116) and (119) lead to the conclusion that

$$
\begin{aligned}
& M(a, b)>I^{5 / 7}(a, b) C^{2 / 7}(a, b), \\
& M(a, b)<I^{\lambda_{2}}(a, b) C^{1-\lambda_{2}}(a, b) .
\end{aligned}
$$

Therefore, Theorem 15 follows from (120) together with the following statements.

(i) If $\alpha_{2}<5 / 7$, then (109) and (110) imply that there exists $\delta_{3} \in(0,1)$ such that $M(a, b)<I^{\alpha_{2}}(a, b) C^{1-\alpha_{2}}(a, b)$ for all $a, b>0$ with $(a-b) /(a+b) \in\left(0, \delta_{3}\right)$.

(ii) If $\beta_{2}>\lambda_{2}$, then (109) and (111) imply that there exists $\delta_{4} \in(0,1)$ such that $M(a, b)>I^{\beta_{2}}(a, b) C^{1-\beta_{2}}(a, b)$ for all $a, b>0$ with $(a-b) /(a+b) \in\left(1-\delta_{4}, 1\right)$.

\section{Acknowledgments}

This research was supported by the Natural Science Foundation of China under Grants 11171307, 61174076, and 61173123 and the Natural Science Foundation of Zhejiang Province under Grants Z1110551 and LY12F02012.

\section{References}

[1] E. Neuman and J. Sándor, "On the Schwab-Borchardt mean," Mathematica Pannonica, vol. 14, no. 2, pp. 253-266, 2003.

[2] J. Chen and B. Hu, "The identric mean and the power mean inequalities of Ky Fan type," Facta Universitatis, no. 4, pp. 1518, 1989.

[3] J. Sándor, "A note on some inequalities for means," Archiv der Mathematik, vol. 56, no. 5, pp. 471-473, 1991.

[4] J. Sándor, "On certain inequalities for means," Journal of Mathematical Analysis and Applications, vol. 189, no. 2, pp. 602606, 1995.

[5] J. Sándor, "On refinements of certain inequalities for means," Archivum Mathematicum, vol. 31, no. 4, pp. 279-282, 1995.

[6] J. Sándor, “Two inequalities for means," International Journal of Mathematics and Mathematical Sciences, vol. 18, no. 3, pp. 621623, 1995.

[7] T. Trif, "On certain inequalities involving the identric mean in $n$ variables," Universitatis Babeş-Bolyai, vol. 46, no. 4, pp. 105-114, 2001.

[8] E. Neuman and J. Sándor, "On the Schwab-Borchardt mean. II," Mathematica Pannonica, vol. 17, no. 1, pp. 49-59, 2006.

[9] L. Zhu, "New inequalities for means in two variables," Mathematical Inequalities \& Applications, vol. 11, no. 2, pp. 229-235, 2008.

[10] L. Zhu, "Some new inequalities for means in two variables," Mathematical Inequalities \& Applications, vol. 11, no. 3, pp. 443448, 2008. 
[11] O. Kouba, "New bounds for the identric mean of two arguments," Journal of Inequalities in Pure and Applied Mathematics, vol. 9, no. 3, article 71, 6 pages, 2008.

[12] M.-K. Wang, Y.-M. Chu, and Y.-F. Qiu, "Some comparison inequalities for generalized Muirhead and identric means," Journal of Inequalities and Applications, vol. 2010, Article ID 295620, 10 pages, 2010.

[13] Y.-M. Chu, M.-K. Wang, and Z.-K. Wang, "A sharp double inequality between harmonic and identric means," Abstract and Applied Analysis, vol. 2011, Article ID 657935, 7 pages, 2011.

[14] Y.-F. Qiu, M.-K. Wang, Y.-M. Chu, and G.-D. Wang, “Two sharp inequalities for Lehmer mean, identric mean and logarithmic mean," Journal of Mathematical Inequalities, vol. 5, no. 3, pp. 301-306, 2011.

[15] S. Gao, "Inequalities for the Seiffert's means in terms of the identric mean," Journal of Mathematical Sciences, vol. 10, no. 1-2, pp. 23-31, 2011.

[16] M.-K. Wang, Z.-K. Wang, and Y.-M. Chu, "An optimal double inequality between geometric and identric means," Applied Mathematics Letters, vol. 25, no. 3, pp. 471-475, 2012.

[17] Y.-M. Chu, S.-W. Hou, and Z.-H. Shen, "Sharp bounds for Seiffert mean in terms of root mean square," Journal of Inequalities and Applications, vol. 2012, article 11, 6 pages, 2012.

[18] Y.-M. Chu and S.-W. Hou, "Sharp bounds for Seiffert mean in terms of contraharmonic mean," Abstract and Applied Analysis, vol. 2012, Article ID 425175, 6 pages, 2012.

[19] Y. M. Li, B. Y. Long, and Y. M. Chu, "Sharp bounds for the Neuman-Sándor mean in terms of generalized logarithmic mean," Journal of Mathematical Inequalities, vol. 6, no. 4, pp. 567-577, 2012.

[20] E. Neuman, "A note on a certain bivariate mean," Journal of Mathematical Inequalities, vol. 6, no. 4, pp. 637-643, 2012.

[21] T. H. Zhao, Y. M. Chu, and B. Y. Liu, "Optimal bounds for Neuman-Sándor mean in terms of the convex combinations of harmonic, geometric, quadratic, and contraharmonic means," Abstract and Applied Analysis, vol. 2012, Article ID 302635, 9 pages, 2012.

[22] Y. M. Chu and B. Y. Long, "Bounds of the Neuman-Sándor mean using power and identric means," Abstract and Applied Analysis, vol. 2013, Article ID 832591, 6 pages, 2013.

[23] J. Sándor, “On the identric and logarithmic means," Aequationes Mathematicae, vol. 40, no. 2-3, pp. 261-270, 1990.

[24] J. Sándor, “On certain identities for means," Universitatis BabeşBolyai, vol. 38, no. 4, pp. 7-14, 1993.

[25] J. Sándor and I. Raşa, "Inequalities for certain means in two arguments," Nieuw Archief voor Wiskunde, vol. 15, no. 1-2, pp. 51-55, 1997.

[26] H. Alzer, "Ungleichungen für Mittelwerte," Archiv der Mathematik, vol. 47, no. 5, pp. 422-426, 1986.

[27] H. Alzer and S.-L. Qiu, "Inequalities for means in two variables," Archiv der Mathematik, vol. 80, no. 2, pp. 201-215, 2003.

[28] Y. M. Chu, M. K. Wang, and S. L. Qiu, "Optimal combinations bounds of root-square and arithmetic means for Toader mean," The Proceedings of the Indian Academy of Sciences, vol. 122, no. 1, pp. 41-51, 2012. 


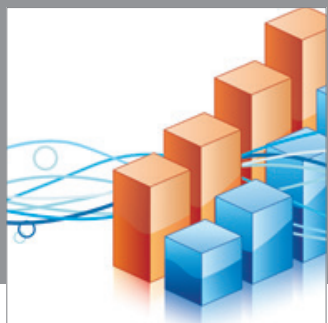

Advances in

Operations Research

mansans

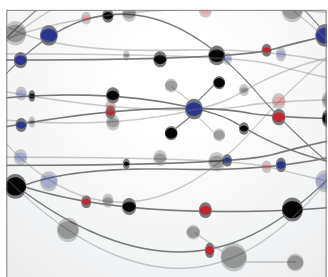

The Scientific World Journal
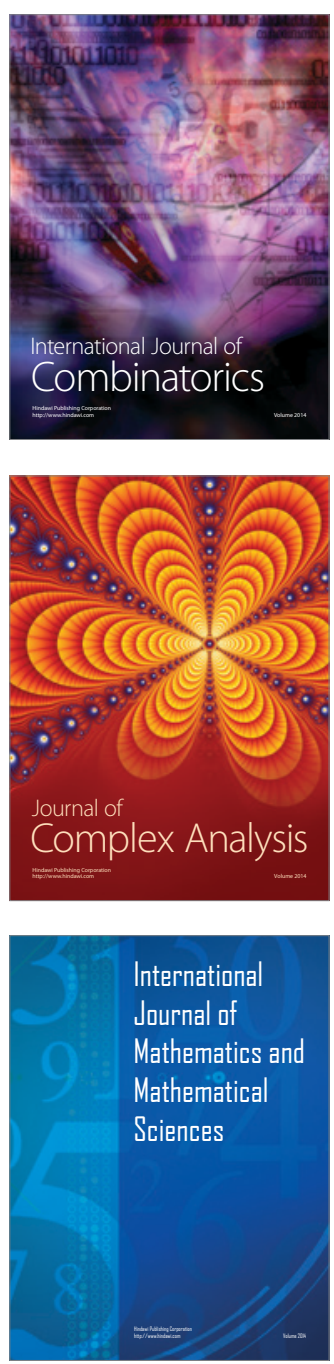
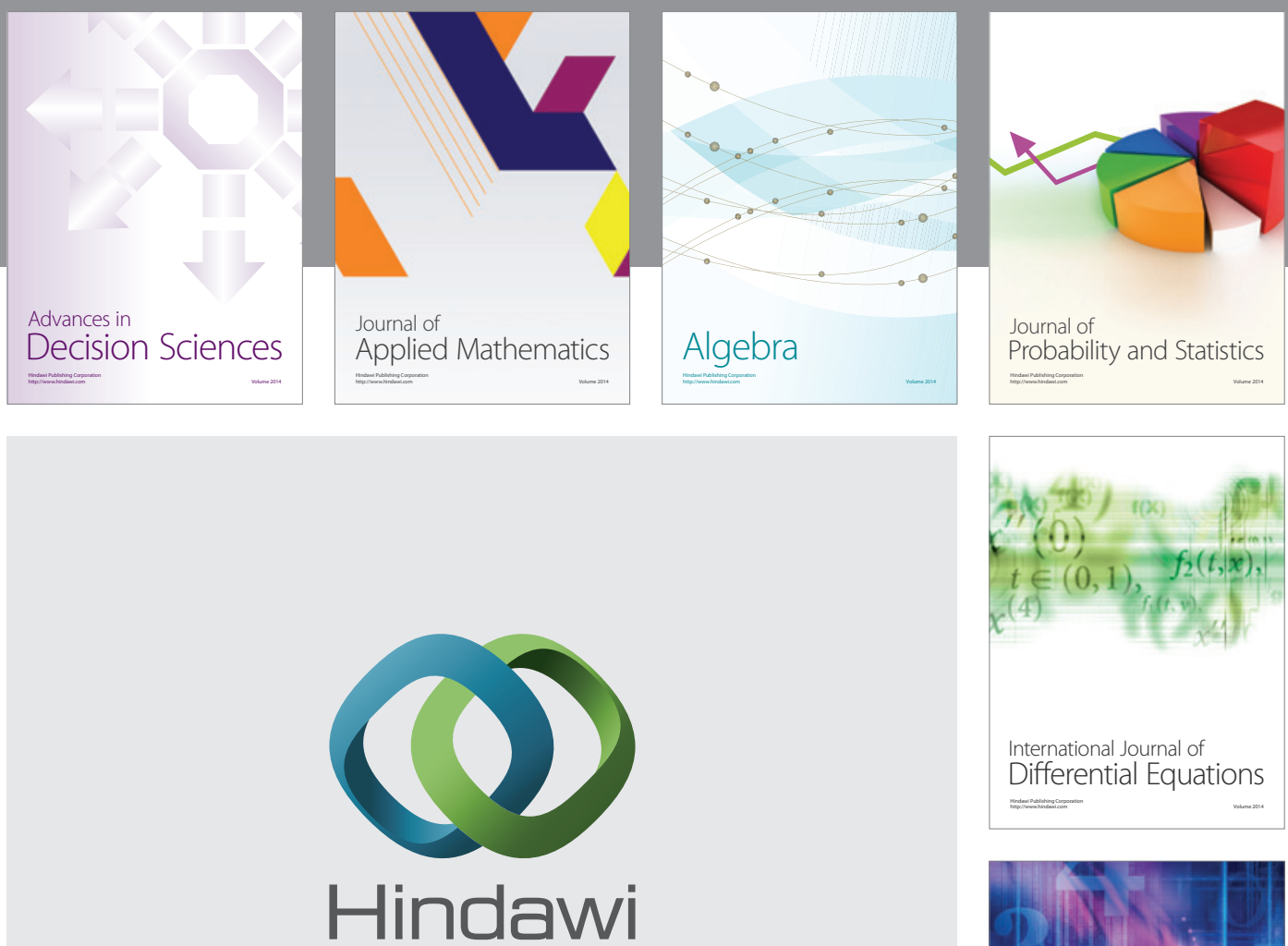

Submit your manuscripts at http://www.hindawi.com
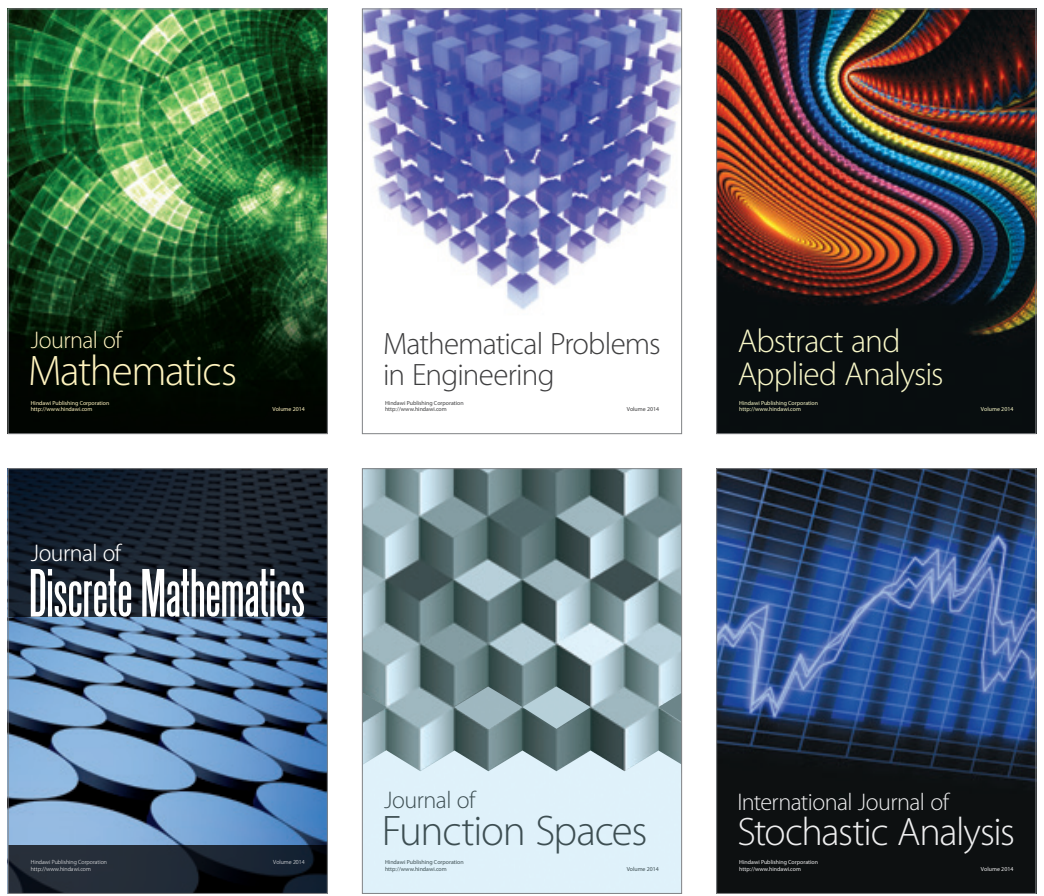

Journal of

Function Spaces

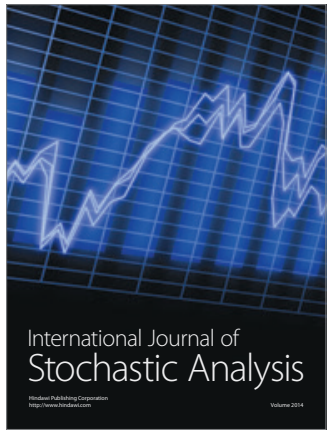

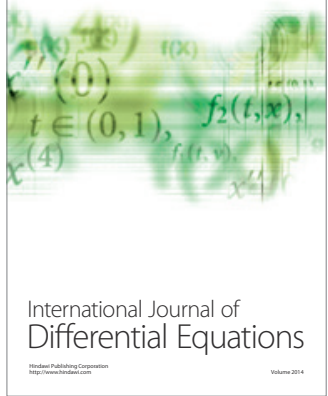
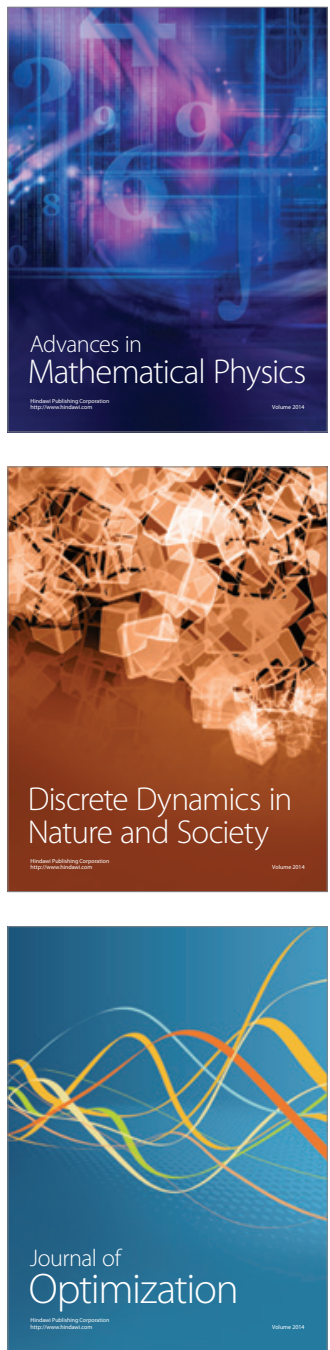